\title{
A one-pot-one-reactant synthesis of platinum compounds at the nanoscale
}

Article

Accepted Version

Stoppiello, C. T., Biskupek, J., Li, Z. Y., Rance, G. A., Botos, A., Fogarty, R. M., Bourne, R. A., Yuan, J., Lovelock, K. R. J., Thompson, P., Fay, M. W., Kaiser, U., Chamberlain, T. W. and Khlobystov, A. N. (2017) A one-pot-one-reactant synthesis of platinum compounds at the nanoscale. Nanoscale, 9 (38). pp. 14385-14394. ISSN 2040-3364 doi: https://doi.org/10.1039/C7NR05976K Available at https://centaur.reading.ac.uk/73605/

It is advisable to refer to the publisher's version if you intend to cite from the work. See Guidance on citing.

Published version at: http://dx.doi.org/10.1039/C7NR05976K

To link to this article DOI: http://dx.doi.org/10.1039/C7NR05976K

Publisher: The Royal Society of Chemistry

All outputs in CentAUR are protected by Intellectual Property Rights law, including copyright law. Copyright and IPR is retained by the creators or other copyright holders. Terms and conditions for use of this material are defined in the End User Agreement. 


\section{CentAUR}

Central Archive at the University of Reading

Reading's research outputs online 


\title{
Nanoscale
}

\section{ARTICLE}

\section{A One-Pot-One-Reactant Synthesis of Platinum Compounds at the Nanoscale}

Received 00th January 20xx, Accepted 00th January 20xx DOI: $10.1039 / \times 0 \times x 00000 x$ www.rsc.org/

\author{
C. T. Stoppiello, ${ }^{a}$ J. Biskupek, ${ }^{b}$ Z. Y. Li, ${ }^{c}$ G. A. Rance, ${ }^{a, d}$ A. Botos, ${ }^{a}$ R. M. Fogarty, ${ }^{e}$ R. A. Bourne, ${ }^{f}$ J. Yuan, ${ }^{g}$

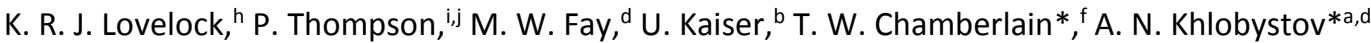

\begin{abstract}
The preparation of inorganic nanomaterials with a desired structure and specific properties requires the ability to strictly control their size, shape and composition. A series of chemical reactions with platinum compounds carried out within the $1.5 \mathrm{~nm}$ wide channel of single-walled carbon nanotubes (SWNTs) have demonstrated the ability of SWNTs to act as both a very effective reaction vessel and a template for the formation of nanocrystals of platinum di-iodide and platinum di-sulphide, materials that are difficult to synthesise in the form of nanoparticles by traditional synthetic methods. The stepwise synthesis inside nanotubes has enabled the formation of Pt compounds to be monitored at each step of the reaction by aberration-corrected high resolution transmission electron microscopy (AC-HRTEM), verifying the atomic structures of the products, and by an innovative combination of fluorescence-detected X-ray absorption spectroscopy (FD-XAS) and Raman spectroscopy, monitoring the oxidation states of the platinum guest-compounds within the nanotube and the vibrational properties of the host-SWNT respectively. This coupling of complementary spectroscopies reveals that electron transfer between the guest-compound and the host-SWNT can occur in either direction depending on the composition and structure of the guest. A new approach for nanoscale synthesis in nanotubes developed in this study utilises the versatile coordination chemistry of Pt which has enabled the insertion of the required chemical elements (e.g. metal and halogens or chalcogens) into the nanoreactor in the correct proportions for the controlled formation of $\mathrm{Ptl}_{2}$ and $\mathrm{PtS}_{2}$ with the correct stoichiometry.
\end{abstract}

\section{Introduction}

The development of new methodologies for the controlled arrangement of molecules and atoms into highly ordered arrays is vitally important for harnessing the functional properties emerging at the nanoscale due to the effects of confinement, which are essential for catalytic, electronic and biomedical applications. The cylindrical internal cavity of single-walled carbon nanotubes (SWNTs) provides an effective template for control of the exact positions and orientations of the molecules and atoms and has been successfully applied to the construction of nanoscale architectures from a variety of metals, organic molecules, ${ }^{1-3}$ and inorganic compounds. ${ }^{4-9} \mathrm{~A}$ striking example of the power of this technique was reported by

a School of Chemistry, University of Nottingham, University Park, Nottingham NG7 2RD, U.K.

${ }^{b}$ Central Facility for Electron Microscopy, Ulm University, Albert-Einstein-Allee 11, Ulm D-89081, Germany.

${ }^{c}$ Nanoscale Physics Research Laboratory, School of Physics and Astronomy, University of Birmingham, Birmingham B15 2TT, U.K.

${ }^{d}$ The Nanoscale and Microscale Research Centre, University of Nottingham University Park, Nottingham NG7 2RD, U.K.

e Department of Chemistry, Imperial College London, London, SW7 2AZ, U.K.

fInstitute of Process Research and Development, School of Chemistry, University of Leeds, Leeds LS2 9JT, U.K.

${ }^{g}$ Department of Physics, University of York, Heslington, York YO10 5DD, U.K.

${ }^{h}$ Inorganic Chemistry Laboratory, University of Oxford, South Parks Road, Oxford, OX 1 $3 Q R$, U.K.

'Department of Physics, University of Liverpool, Liverpool L69 7ZE, U.K.

iXMaS CRG, European Synchrotron Radiation Facility, 38043 Cedex, Grenoble, France.

Electronic Supplementary Information (ESI) available: See DOI: 10.1039/x0xx00000x
Chuvilin et al. who demonstrated that sulfur-terminated graphene nanoribbons, practically impossible to fabricate by synthetic chemistry routes, can be spontaneously formed from inexpensive, readily available precursors, such as tetrathiafulvalene, in one step within SWNTs. ${ }^{10,11}$ More recently, very narrow double-walled carbon nanotubes have been demonstrated as effective containers for templating the important, but still elusive, allotrope of carbon, carbyne. ${ }^{12}$ Shi et al. demonstrated that at high temperature the host nanotube can template the formation of this new allotrope of carbon and protect it from undesired side reactions, thus paving the way for the first bulk production of carbyne. These examples clearly demonstrate the intrinsic ability of the SWNTs to direct chemical reactions and control the dimensionality of the products formed inside nanotubes, where the product structure is crucially determined by the internal diameter of the nanotube.

Low-dimensional inorganic materials, including transition metal chalcogenides, are currently of particular interest due to the fact that their semiconducting electronic properties can be precisely tuned by shaping their structures into nanoribbons, in an analogous fashion to graphene. ${ }^{13}$ While the host-nanotube can control the dimensions of the inorganic structure, control of the stoichiometry is typically achieved by immersing open SWNTs into a molten inorganic compound with the desired, predetermined chemical composition, so that the nanotube becomes filled with cations and anions in the correct ratio due to capillary forces. A now ubiquitous technique, it has led to the encapsulation of many inorganic compounds, such as lanthanide halides, ${ }^{14}$ potassium iodide, ${ }^{15}$ cobalt iodide, ${ }^{16}$ tin telluride, ${ }^{17}$ and barium di-iodide, ${ }^{6}$ yielding nanostructures with well-defined composition and morphology, and physical properties 
often different to those found in the bulk. However, the need to melt inorganic compounds in this method imposes severe limitations on the choice of encapsulated materials, as many important inorganic materials, such as metal sulfides, decompose before melting or melt at extremely high temperature, e.g. the melting point of $\mathrm{MoS}_{2}$ is $1185^{\circ} \mathrm{C}$.

The direct synthesis of inorganic compounds inside nanotubes can offer an attractive alternative which may significantly broaden the scope of inorganic compounds that are confined to the nanoscale. The nanotube in this case acts simultaneously as a nanoscale reactor and a template for controlling the dimensions of inorganic structures. Inorganic synthesis inside nanotubes has been successfully applied by Eliseev et al. to the synthesis of CdS@SWNT by treating pre-encapsulated $\mathrm{Cdl}_{2}$, inserted into nanotubes from the molten phase, with sulfur at high temperature. ${ }^{18}$ This elegantly avoids the problem that bulk CdS has a melting point of $1750{ }^{\circ} \mathrm{C}$, precluding the molten phase filling of this material. Similarly, the formation of low-dimensional $\mathrm{MS}_{2}\left(\mathrm{M}=\mathrm{Mo}^{19} \mathrm{~W}^{20}\right)$ in multi-walled carbon nanotubes has been demonstrated by Wang et al. via the decomposition of molecular complexes highlighting the further potential for tailoring bespoke semiconducting inorganic materials with nanoscale precision. Recently, we demonstrated the formation of $\mathrm{MS}_{2} @ S W N T(\mathrm{M}=\mathrm{Mo}, \mathrm{W})$ via a metal-iodide intermediate, and showed that the SWNT acts not only as a template to restrict the morphology of the $\mathrm{MS}_{2}$, but also as a reservoir of electrons to stabilise the reaction intermediate. ${ }^{21}$

In this study, we evaluate carbon nanotubes as nanoreactors in stepwise and one-pot inorganic syntheses, highlighting the advantages and limitations of the different approaches. Using the compounds of platinum as models, we outline a new strategy for the fabrication of inorganic nanomaterials with controlled structure and composition. Conducting the chemical transformations inside the host-nanotube allows for the structural determination of the reaction products with atomic precision by aberration-corrected high resolution transmission electron microscopy (AC-HRTEM) and aberration-corrected scanning transmission electron microscopy (AC-STEM), while our unique approach based on cross-correlation of fluorescence-detected X-ray absorption spectroscopy (FD-XAS) and Raman spectroscopy applied at each stage of inorganic synthesis in carbon nanotubes enables determination of the exact oxidation state of the metal (Pt) in inorganic compounds formed inside nanotubes and the vibrational properties of the host-nanotube respectively. This methodology enables changes in host-guest interactions from one stage of the inorganic synthesis to another to be monitored by multiple techniques for the first time, providing a systematic understanding of the electron transfer between the nanoreactor and encapsulated compound during all stages of the formation process.

\section{Results and discussion}

The stepwise formation of inorganic materials in SWNTs can be achieved by selecting an appropriate metal precursor, i.e. small volatile metal complexes consisting of the required metallic element for the desired inorganic material, and inserting them into the nanotube (figure 1a), followed by thermal treatment to remove the volatile ligand from the nanotube cavity (figure $1 \mathrm{~b}$ ), with the sequential addition of a non-metallic element which reacts with the metal to form the desired inorganic compound. If necessary, more reaction steps can be added to convert the inorganic compound to another product (figure 1d).

To explore and evaluate this approach for inorganic synthesis in SWNTs, platinum acetylacetonate, $\mathrm{Pt}(\mathrm{acac})_{2}$ was inserted into SWNTs from the gas phase, with the excess removed from the exterior of SWNTs by applying a selective $\mathrm{CH}_{2} \mathrm{Cl}_{2}$ washing technique, ${ }^{22}$ leaving only $\mathrm{Pt}(\mathrm{acac})_{2}$ molecules encapsulated within the nanotube, Pt(acac) $)_{2} @ S W N T$. The $L_{\| I}$ edge of Pt (measured in FD-XAS) can be used as a probe of oxidation state, this edge results from excitations between $2 p_{3 / 2}$ and $5 d$ states. Higher Pt oxidation states (lower electron density) stabilise $2 \mathrm{p}_{3 / 2}$ orbitals, and hence lead to larger $P t L_{\| I}$ edge energies. ${ }^{23}$ Furthermore, the characteristic $G$ band, ubiquitously observed in the Raman spectra of graphitic nanostructures, and caused by vibrations of carbon atoms of $E_{28}$ symmetry in an extended lattice, is sensitive to strain and curvature of the nanotube, and can be used to measure any electron transfer from the nanotube to guest molecules. ${ }^{24} \mathrm{~A}$ red shift is indicative of electron transfer from the intercalant to the SWNT, and a blue shift is indicative of electron transfer from the SWNT to the intercalant.

FD-XAS of Pt(acac) $)_{2} @ S W N T$ shows a slight increase in the Pt LIII edge energy relative to that of a $\mathrm{Pt}(\mathrm{acac})_{2}$ control sample (supporting information, table $\mathrm{S}-1$ ) indicating a small reduction of electron density on the metal centre of $\mathrm{Pt}(\mathrm{acac})_{2}$ inside the nanotubes (figure 2a); however, Raman spectroscopy (figure 2b) shows no shift in the G-band of the SWNT suggesting little or no charge transfer between the guest and the nanotube upon encapsualtion. ${ }^{25}$ Heating $\mathrm{Pt}(\mathrm{acac})_{2} @ S W N T$ under argon at $500{ }^{\circ} \mathrm{C}$ for one hour resulted in the decomposition of $\mathrm{Pt}(\mathrm{acac})_{2}$ and the release of metallic $\mathrm{Pt}$ into the SWNT cavities in the form of cylindrical nanoparticles with an average length of $1.5 \mathrm{~nm}$ and diameter of $1.0 \mathrm{~nm}$ as confirmed by TEM imaging (figure $2 c$ inset, see $\mathrm{SI}$ file section $3 \mathrm{~d}$ for AC-HRTEM images). The formation of small metallic nanoparticles rather than a continuous metal nanowire is determined by the limited number of metal atoms available in the nanotube, the stabilising effect of the nanotube and the release of gaseous ligand fragments upon heating, as was reported previously for $\mathrm{W}, \mathrm{Re}$ and Os nanoparticles formed

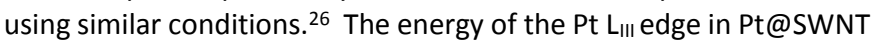
is increased significantly relative to bulk metallic platinum.
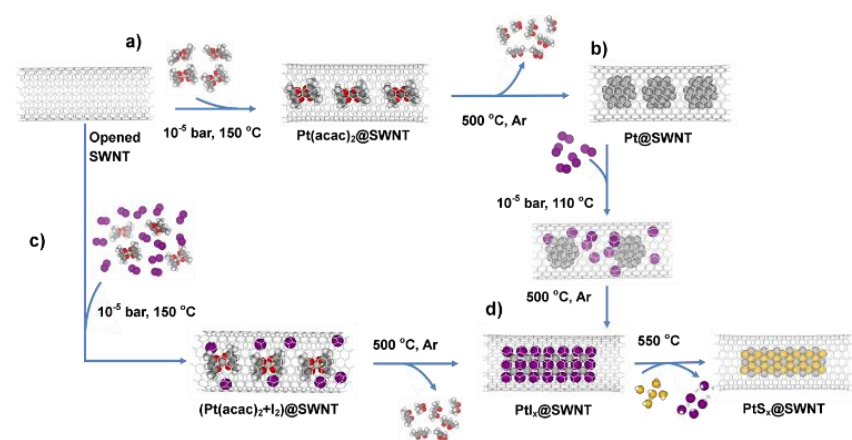

Figure 1: Schematic illustration depicting how inorganic materials can be formed using nanotubes as templates; a) the encapsulation of a selected metal precursor for the desired metallic element followed by thermal decomposition to form metal nanoparticles@SWNT; b) the addition of the second element by adding a second precursor followed by chemical reaction to form the inorganic material; c) the formation of inorganic materials using a one-step filling process in which both precursors, metallic and non-metallic are added at the same time followed by chemical reaction; d) the transformation of one encapsulated inorganic material to another by treatment with a suitable reagent inside the nanotube. 
a)

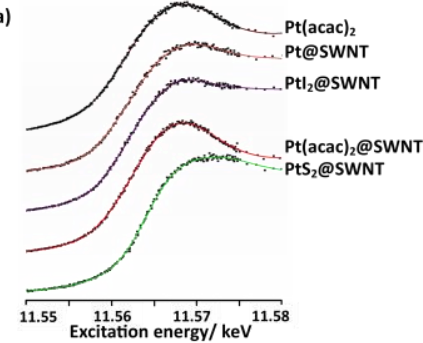

c)

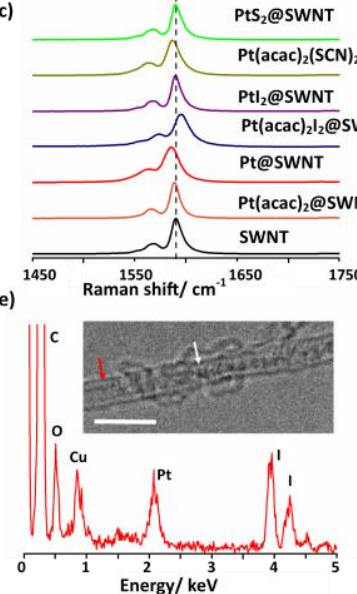

b)

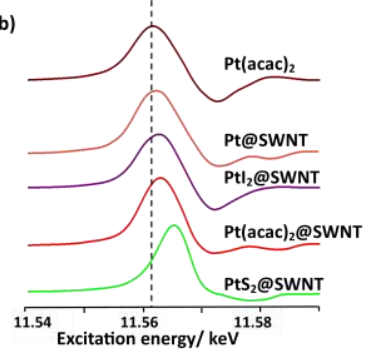

d)

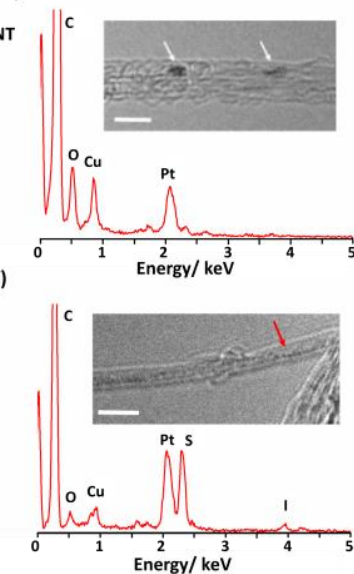

Figure 2: a) FD-XAS spectra showing the Pt $L_{\text {III }}$ edge of materials in SWNTs prepared using a stepwise approach compared to that of bulk Pt(acac) 2. A smoothed line is overlaid on the raw data (black) for each material; b) the derivative of the FD-XAS spectra shown in (a); the dashed line denotes the Pt $L_{\text {III }}$ edge energy of bulk Pt(acac) and shows how this energy varies for different Pt materials within the SWNT; c) Raman spectra showing how the $G$ band of the nanotube shifts depending on the encapsulated material. The dashed line denotes the position of $G$ band of the empty SWNT for reference, the encapsulation of molecular species (Pt complexes or 12) leads to electron transfer between the nanotube and quest; however, the encapsulated $P t X_{2}(X=I$ or $S)$ compounds exhibit no change in G-band meaning that no charge transfer is taking place. d) an EDX spectrum of Pt@SWNT, a low magnification TEM image (the white arrows showing Pt) of the sample is shown inset. The $O$ is from defects in the SWNT; e) an EDX spectrum of PtI $2 @ S W N T$ formed using the one-pot method. An excess of iodine is present in the sample. Inset is a TEM image showing clusters of Pt atoms (white arrow) and I atoms (red arrow) within the SWNT; and f) an EDX spectrum of PtS $@$ @SWNT formed from the treatment of PtI $2 @ S W N T$ with $\mathrm{H}_{2} \mathrm{~S}$. Traces of iodine are still present in the sample. Inset is a TEM image of PtS ${ }_{2} @ S W N T$ formed by the transformation of PtI $2 @ S W N T$. In agreement with the EDX spectrum, excess iodine is present (red arrow); (the scale bars are all $2 \mathrm{~nm}$, all EDX spectra were recorded at $100 \mathrm{keV}$ primary electron energy and integrated over a small bundle of nanotubes.

N.B. Cu peaks are due to the TEM specimen grid.

Simultaneously a small but measurable red shift of the $\mathrm{G}$ band in the Raman spectrum of the host-nanotubes, relative to empty nanotubes is observed, confirming that some electron transfer takes place from metallic Pt to SWNT in the Pt@SWNT material, which correlates with the FD-XAS (table 1). An alternative explanation is the formation of $\mathrm{PtO}_{2}$, but the metallic nature of the Pt nanoparticles, rather than Pt carbide or oxides phases was also verified by carefu comparison of the interatomic spacings in the individual nanoparticles ( $\mathrm{d}_{\mathrm{pt} \text {-Pt }}=\mathrm{ca} .0 .26 \mathrm{~nm}$ ) observed in AC-HRTEM which are closer to Pt metal in a face centred cubic crystal (SI file section $3 \mathrm{~d}$ ).

In the next step, Pt@SWNT was treated with iodine vapour (figure 1b) leading to the direct synthesis of platinum iodide inside nanotubes, $\mathrm{Ptl}_{2} @ S W N T$, from the constituent elements (figure $2 \mathrm{~d}$

inset). Raman spectroscopy shows that the G-band of the hostnanotube in $\mathrm{PtI}_{2} @ S W N T$ returns to a similar position to that of empty SWNTs, and FD-XAS shows that the energy of the Pt edge of $\mathrm{Ptl}_{2} @ S W N T$ is similar to that of known Pt" complexes (table 1), with both methods agreeing that no electron transfer takes place between the nanotube and charge-neutral compound $\mathrm{Ptl}_{2}$, which contrasts with the observed Pt to SWNT electron transfer in Pt@SWNT as described above, or SWNT to iodine electron transfer described for $\mathrm{I}_{2} @ S W N T$ in the literature. ${ }^{25}$ The addition of $\mathrm{H}_{2} \mathrm{~S}$ to $\mathrm{Ptl}_{2} @ S W N T$ led to the transformation of platinum iodide to the platinum sulfide compound PtS $\mathrm{P}_{2} @ \mathrm{SWNT}$ (figure 1d) which in a similar fashion to the iodide exhibits no electron transfer with the nanotube as the position of the G-band observed in the Raman spectrum of PtS ${ }_{2} @ S W N T$ coincides with that of empty SWNTs. This is in agreement with FD-XAS showing a Pt excitation edge energy similar to that of known $\mathrm{Pt}^{\mathrm{IV}}$ complexes (table 1), thus confirming a charge-neutral $\mathrm{PtS}_{2}$ material inside the nanotube. This series of chemical transformations inside carbon nanotubes clearly illustrates that stepwise inorganic synthesis involving transformations of a metal complex to metal nanoparticles, to metal iodide and to metal sulfide can be achieved in SWNTs with each stage verified by TEM, as well as FD-XAS and Raman spectroscopy revealing the nature of the interactions between the inorganic compounds and the host-nanotube.

In a separate experiment, both $\mathrm{Pt}(\mathrm{acac})_{2}$ and $\mathrm{I}_{2}$ were encapsulated in SWNTs simultaneously, from the gas phase followed by heat treatment of the encapsulated molecules (Pt $\left.(\mathrm{acac})_{2}+\mathrm{I}_{2}\right) @ S W N T$ that triggers a chemical reaction between $\mathrm{Pt}(\mathrm{acac})_{2}$ and $\mathrm{I}_{2}$ to form $\mathrm{Ptl}_{2} @ S W N T$ in one step, which is analogous to a popular one-pot synthetic approach in preparative chemistry in the absence of SWNTs. This platinum iodide appears to be identical to the same compound formed in the stepwise process above, which clearly indicates the advantages of the one-pot synthesis in nanotubes, allowing for the bypass of several stages of the stepwise approach, i.e. $\mathrm{Pt}(\mathrm{acac})_{2}$ insertion and thermal decomposition, followed by $I_{2}$ insertion into SWNTs. While both of these approaches appear to be effective in controlling the dimensions of inorganic nanostructures, being strictly defined by the diameter of SWNTs, detailed analysis of the elemental composition of the inorganic products reveals a problem of stoichiometry control. Unlike organic molecular compounds, inorganic materials have a capacity for nonstoichiometric ratios of the constituent elements, which is evident from energy dispersive X-ray (EDX) analysis indicating that the materials synthesised inside nanotubes in both approaches have a non-stoichiometric ratio of metal and halogen/chalcogen, (SI file, section 5). Specifically, the content of iodine in $\mathrm{Ptl}_{2} @ S W N T$ appears to be consistently higher than predicted for $\mathrm{Ptl}_{2}$ (figure $2 \mathrm{~d}$ ), and traces of iodine still remain present in the nanotubes even after conversion of $\mathrm{PtI}_{2} @ S W N T$ to $\mathrm{PtS}_{2} @ S W N T$ (figure 2e). This observation clearly highlights the challenge of controlling the correct proportions of reactants in nanotubes: $I_{2}$ is more volatile than $\mathrm{Pt}(\mathrm{acac})_{2}$ and has a lower vapour pressure which means that it will always be loaded into the nanotube in excess of the Pt precursor, thus making the removal of extra iodine difficult, even after platinum iodide is transformed into platinum sulfide (figure 2e). As even a small deviation from the exact stoichiometric composition causes drastic effects in the physiochemical properties of inorganic materials, such as in the case of diamond doping with $\mathrm{N}$, or boron nitride doping with $\mathrm{C}$, effective control of the ratios of the elements is of paramount importance in inorganic synthesis at the nanoscale. ${ }^{27,28}$ 
Table 1: Shifts of the Pt $L_{111}$ FD-XAS edge energy for the different platinum materials in SWNTs with resepect to (w.r.t.) their free state, and shifts in the G-band in the Raman spectra of nanotubes filled with different platinum materials w.r.t. that of empty SWNTs.

\begin{tabular}{|c|c|c|c|}
\hline Material & $\begin{array}{l}\text { Shift of FD-XAS edge energy w.r.t. free } \\
\text { compound/eV }\end{array}$ & $\begin{array}{l}\text { Shift of G-band w.r.t. empty } \\
\text { SWNT/cm-1 }\end{array}$ & $\begin{array}{c}\text { Direction of electron } \\
\text { transfer }\end{array}$ \\
\hline Pt(acac) $)_{2} @ S W N T$ & +0.5 & 0 & No transfer observed \\
\hline Pt@SWNT & +1.0 & -4 & $\mathrm{Pt} \rightarrow \mathrm{SWNT}$ \\
\hline $\mathrm{Pt}(\mathrm{acac})_{2} \mathrm{l}_{2} @ S W N T$ & -1.5 & +6 & $\mathrm{Pt} \leftarrow$ SWNT \\
\hline PtI ${ }_{2} @ S W N T$ & +0.3 & 0 & No transfer observed \\
\hline $\mathrm{Pt}(\mathrm{acac})_{2}(\mathrm{SCN})_{2} @ \mathrm{SWNT}$ & -0.6 & -5 & $\mathrm{Pt} \rightarrow \mathrm{SWNT}$ \\
\hline Pts ${ }_{2} @ S W N T$ & $\begin{array}{l}+0.4 \text { (relative to another known } \mathrm{Pt}^{\mathrm{IV}} \\
\text { compound) }\end{array}$ & 0 & No transfer \\
\hline
\end{tabular}

A solution to this challenge can be found by designing a molecular precursor where the elements are present in the desired stoichiometric ratio for a specific inorganic product, which can then be encapsulated and converted into the inorganic material in SWNTs in a one-pot-one-reactant synthesis (figure 3 ). The advantage of such an approach is that it involves a single step and the stoichiometric ratio of the metal and halogen/chalcogen components in the material formed can be carefully tuned. The use of coordination compounds of transition metals offers excellent potential for the control of the composition and stoichiometry of the reactants. For example, the coordination environment of four-coordinate $\mathrm{Pt}$ complexes, such as $\mathrm{Pt}(\mathrm{acac})_{2}$, can be expanded to six-coordinate by appending two extra ligands to the axial positions, which can carry the correct amounts of the desired non-metallic element. For example, the addition of two iodide or two thiocyanate ligands that occupy the two axial positions in the octahedral complex of Pt allows fixing of the ratio of metal to halogen or chalcogen to $1: 2$ thus ensuring the correct stoichiometry of the inorganic compound formed inside the nanotubes (figure 3). To illustrate the principle of one-pot-one-reactant synthesis in nanoreactors, we prepared the molecular precursors trans-bis(acetylacetonato)di-iodoplatinum and bis(acetylacetonato)di-thiocyanatoplatinum containing Pt:I (1:2) and $\mathrm{Pt}: S(1: 2)$ respectively by reacting $\mathrm{Pt}(\mathrm{acac})_{2}$ with $\mathrm{I}_{2}$ to give $\mathrm{Pt}(\mathrm{acac})_{2} \mathrm{I}_{2}$, and then reacting $\mathrm{Pt}(\mathrm{acac})_{2} \mathrm{I}_{2}$ with $\mathrm{AgSCN}$ to afford $\mathrm{Pt}(\mathrm{acac})_{2}(\mathrm{SCN})_{2}$, using previously reported methods (scheme 1). ${ }^{29,30}$

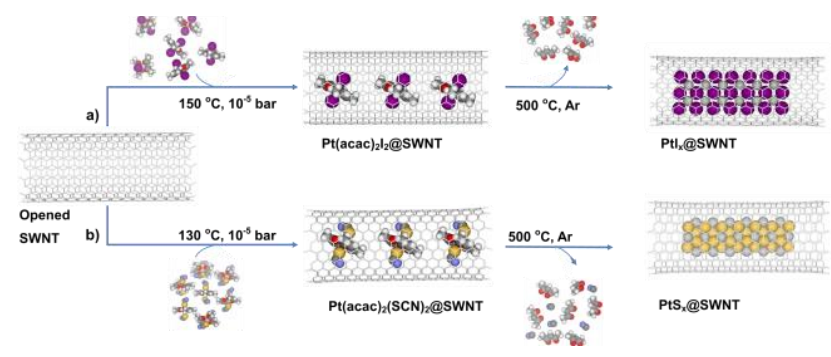

Figure 3: Schematic illustration depicting how inorganic materials can be formed using nanotubes as templates. The formation of a) PtI @SWNT; and b),PtS ${ }_{2} @ S W N T$ are shown using our methodology. In general a molecular precursor which contains all of the required elements, in the correct stoichiometry for the desired inorganic material, is encapsulated. The thermal treatment of the resultant precursor then leads to decomposition, chemical reaction to form the inorganic material in the nanotubes and expulsion of any volatile by-products into the gas phase.

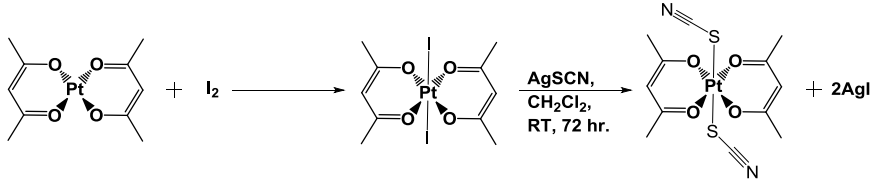

Scheme 1: The formation of $\mathrm{Pt}(\mathrm{acac})_{2} \mathrm{l}_{2}$ and $\mathrm{Pt}(\mathrm{acac})_{2}(\mathrm{SCN})_{2}$ from $\mathrm{Pt}(\mathrm{acac})_{2}$.

The resultant $\mathrm{Pt}(\mathrm{acac})_{2} \mathrm{X}_{2}(\mathrm{X}=\mathrm{I}$, figure $3 \mathrm{a} ; \mathrm{X}=\mathrm{SCN}$, figure $3 \mathrm{~b})$ compounds were added to SWNTs and sealed in an ampoule under vacuum $\left(10^{-5}\right.$ bar $)$. Heating at a temperature slightly above the sublimation points of the platinum complexes allowed the precursor molecules to penetrate the open ends of the nanotubes and become encapsulated. The excess material was removed from the nanotube surface by washing with $\mathrm{CH}_{2} \mathrm{Cl}_{2}$, leaving only molecules inserted in the nanotubes. Interestingly, the encapsulation of Pt(acac) $)_{2} @$ @SWT led to a significant reduction in the absorption energy of the Pt $\mathrm{L}_{\text {III }}$ edge indicative of an increase of electron density onto the Pt centre as compared to free $\mathrm{Pt}(\mathrm{acac})_{2} \mathrm{I}_{2}$ (figure $4 \mathrm{a}$ ), which is complemented by a significant blue shift of the Raman G-band of the host SWNT (figure $2 \mathrm{~b}$ ), implying electron transfer from the nanotube to the guest molecule which has not been observed for any other $\mathrm{Pt}$ compounds in this study. In contrast, Pt $(\mathrm{acac})_{2}(\mathrm{SCN})_{2} @ S W N T$ showed a decrease in the Pt edge energy in the FD-XAS (figure $4 \mathrm{~b}$ ) and $a$ red shift of the nanotube $G$ band (figure $2 b$ ), indicating that the electron is transferred in the opposite direction to the previous complex, i.e. from $\mathrm{Pt}(\mathrm{acac})_{2}(\mathrm{SCN})_{2}$ to SWNTs. A simple thermal treatment led to the facile transformation of the molecular precursors to the desired inorganic structures, Pt| ${ }_{2} @ S W N T$ and $\mathrm{PtS}_{2} @ S W N T$ (figure 3) with the nearly stoichiometric 1:2 metal to halogen/chalcogen composition, as confirmed by EDX spectroscopy (figures $4 c$, d, SI section S5).

While the new one-pot-one-reactant approach clearly enabled a precise control of the stoichiometry, it is essential to verify the atomic structure of the inorganic compounds formed in this method, to ensure that the new molecular precursors still deliver inorganic materials with well-defined atomic structures. AC-HRTEM is one of the most direct tools for the structural characterisation of inorganic materials formed within SWNTs, as the precise atomic composition and any distortions in the crystal lattice can be clearly visualised to determine the effects of nanoscale confinement. ${ }^{20,31,32}$ AC-HRTEM of the Pt1 $2 @ S W N T$ sample prepared by the one-pot-one-reactant approach revealed high filling of $\mathrm{Ptl}_{2}$ within the SWNTs (figure $5 \mathrm{a}$ ) in which the entire channel was filled with the crystalline inorganic material. 

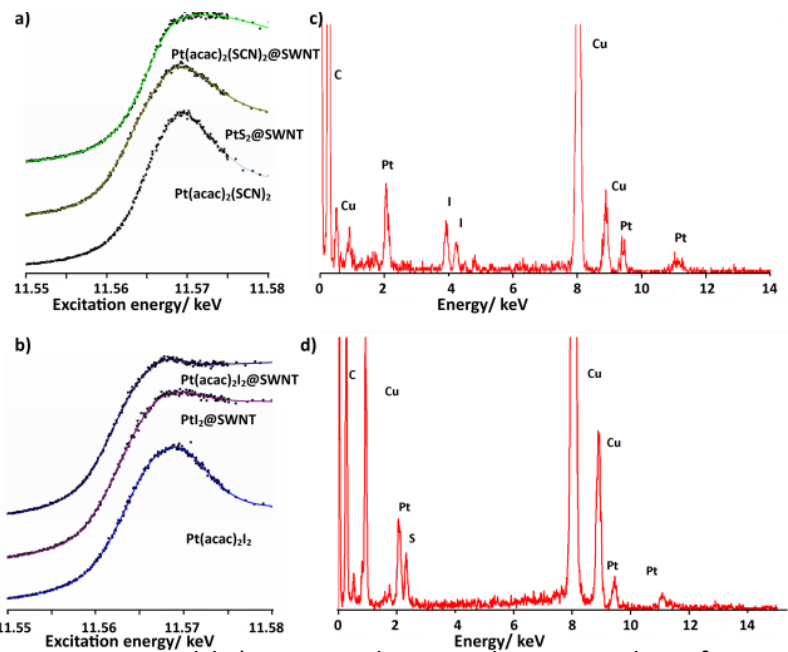

Figure 4: $a$ and $b$ ) FD-XAS showing the Pt $L_{\text {III }}$ edge of materials formed in SWNTs, using molecular precursors containing suitable amounts of material for the formation of PtI $@$ @SWN (a) and PtS ${ }_{2} @ S W N T$ (b), The raw data (black) has been overlaid on the smoothed versions; EDX spectra recorded at $100 \mathrm{keV}$ primary electron energy and integrated over a small bundle of nanotubes showing c), PtI ${ }_{2} @ S W N T$; and d), PtS ${ }_{2} @ S W N T$, both formed via the thermal decomposition of molecular precursors containing the desired stoichiometric ratios of the two elements inside SWNTs. The spectra confirm the successful formation of the inorganic materials inside the nanotubes in each case (N.B. Cu peaks are due to the TEM specimen grid).

In a representative example, an individual filled nanotube with a diameter of ca $1.52 \mathrm{~nm}$, that corresponds to that of a $(11,11)$ SWNT (figure 5a), is observed to contain a quasi-one-dimensional $\mathrm{Ptl}_{2}$ crystal characterised by a close-packed cubic lattice (figure $5 c$ ). In the bulk, $\mathrm{PtI}_{2}$ crystals pack in the $P 2(1) / c$ group of symmetry with each Pt six-coordinate and each I three-coordinate. ${ }^{33}$ Such an arrangement of atoms gives rise to a monoclinic lattice with a unit cell $6.59 \AA$ × $8.71 \AA$ × $6.89 \AA$, which although could fit into the cavity of a SWNT, leads to a large void inside the SWNT, and so the packing of the $\mathrm{Ptl}_{2}$ changes to better fit into the SWNT and minimise the empty space. Therefore, the stoichiometry of Pt and I is maintained, but the arrangement of atoms changes as compared to that in bulk $\mathrm{PtI}_{2}$. Specifically, while the interatomic Pt-I distances remain the same as in the bulk crystal and Pt is still six-coordinate, the iodine atoms change coordination numbers to range from 1 to 6 , depending on their position within the new unit cell (SI section 4, figure S-12). This allows a more compact structure with a more suitable unit cell commensurate with the size of SWNT. AC-HRTEM imaging and TEM image simulation reveal a type of lattice that is closer to a cubic rather than monoclinic (figure 5b), with highcoordinated I-atoms in the middle of the structure and lowcoordinated I-atoms located between in the space between Pt and SWNT wall, such that the overall ratio of $\mathrm{Pt}$ to $\mathrm{I}$ is $1: 2$, the same as in the bulk crystal.

Lattice measurements in experimental AC-HRTEM images showed regular spacings between atomic positions in the direction parallel to the nanotube of $0.39 \mathrm{~nm}$, compared to a distance of $0.381 \mathrm{~nm}$ between I atoms in the asymmetric unit of bulk $\mathrm{PtI}_{2}$. Such a level of distortion in inorganic structures is consistent with the literature and is a result of the confined lattice adapting to the nanotube channel in order to increase van der Waals interactions with the nanotube sidewalls. ${ }^{6,14,32}$ Similarly, there appears to be a slight increase in the observed diameter of the SWNT due to an elliptical distortion of the nanotube channel to accommodate the $\mathrm{Ptl}_{2}$ species (figure $5 \mathrm{~d}$ and e)
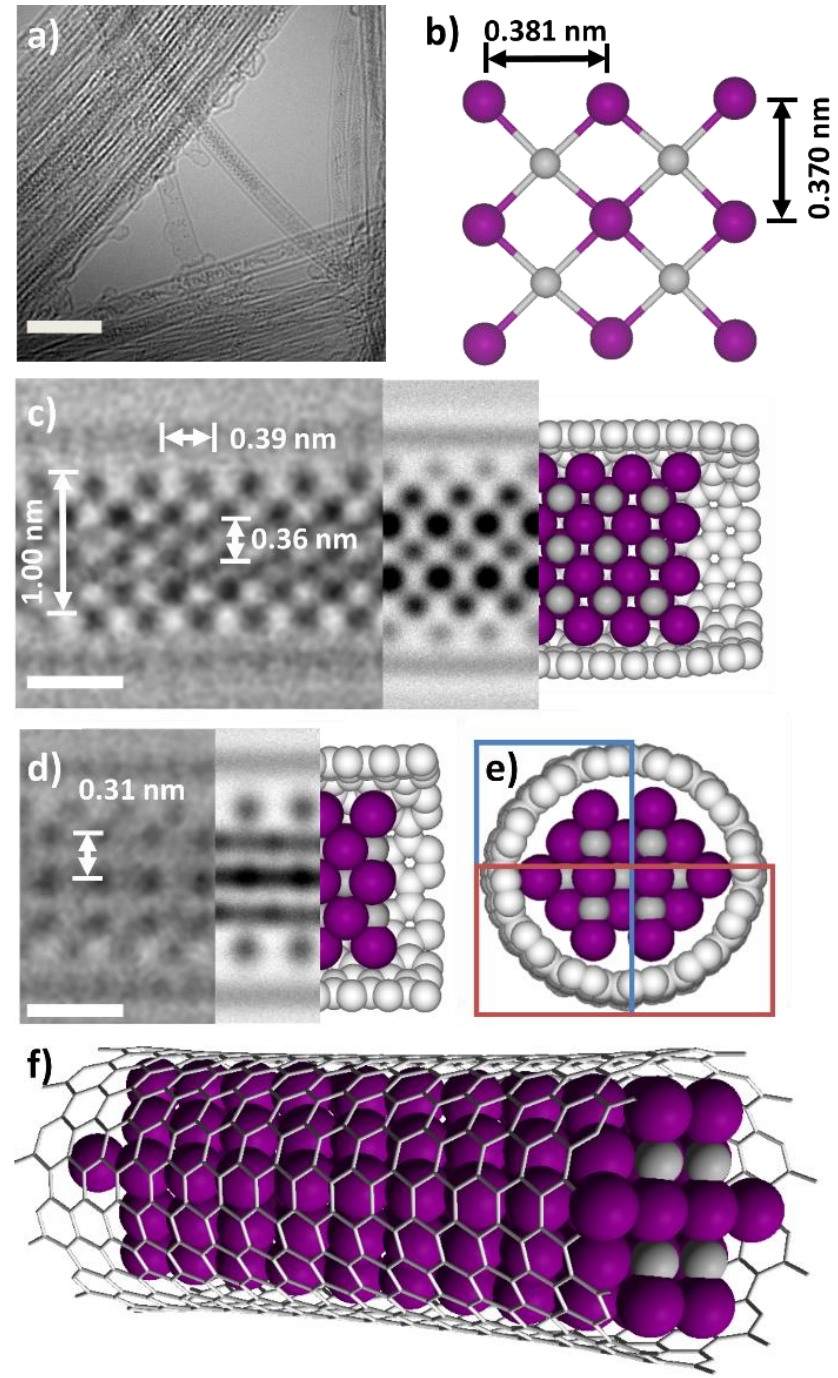

Figure 5: a) An AC-HRTEM micrograph showing a bundle of Pt1 $2 @ S W N T$ in which the majority of the SWNTs are highly filled with inorganic material, the scale bar is $5 \mathrm{~nm}$; b) An extended portion of the crystal structure of $\mathrm{PtI}_{2}$ derived from the asymmetric unit of bulk PtI 2 with key atomic distances shown; the grey and purple spheres represent platinum and iodine atoms respectively; c) an AC-HRTEM micrograph showing a small section of a $1.52 \mathrm{~nm}$ SWNT containing PtI 2 material and the corresponding atomic distances to the structure in b) are shown. A HR-TEM simulation $(80 \mathrm{kV})$ showing a projection of how a portion of the derived Pt/ structure could fit inside an $(11,11)$ SWNT, complemented by the cross-section of computational simulation to show its shape in the nanotube, the scale bar is $0.5 \mathrm{~nm}$; d) an AC-HRTEM of the same section of nanotube with the material in a different orientation is shown, along with an AC-HRTEM simulation (80 kV) showing a projection of the model from (c) when rotated $90^{\circ}$ parallel to the nanotube axis, and a computational simulation of this model to show its shape in the nanotube, the scale bar is $0.5 \mathrm{~nm}$; e) a simulation to show how the crystal fragment would appear when looking down the nanotube. The red and blue boxes represent the sections of the tube visible in figures $c$ and $d$ respectively; $f$ ) $a$ geometry optimised model showing how the proposed $\mathrm{PtI}_{2} \mathrm{crystal}$ would fit into a $(11,11)$ SWNT.

which has also been reported previously for such systems. ${ }^{16}$ Computational modelling was utilised to generate a 3D representation of $\mathrm{Ptl}_{2} @ S W N T$ (figure $5 f$ ) to enable comparison with experimental AC-HRTEM images. AC-HRTEM images were then calculated by a TEM image multislice simulation package (QSTEM) using a model of a $(11,11)$ SWNT filled with a structure derived crystal 
fragment. The simulated images ( $80 \mathrm{kV}$ voltage, Scherzer conditions for under focus, spherical aberration parameter $C_{s}=10 \mu \mathrm{m}$ ) are in good agreement with the experimentally obtained images (figure $5 c$, $d$, and e). The presence of terminal iodine atoms within the structure is supported by local probe, quantitative EDX analysis which reveals a Pt:I ratio of 1:2.11, see SI file section 5 .

In bright-field AC-HRTEM images (figure 5) the observed contrast is determined by a complex mixture of dynamic effects depending on thickness and crystallinity of the structures as well as the actual focus conditions and the atomic number of the individual atoms, which makes the discrimination between different elements, particularly those similar in atomic number, complex. As a consequence, without quantitative image simulation, bright-field TEM is not a very efficient method for distinguishing between two heavy elements such as $\mathrm{Pt}$ $(Z=78)$ and I ( $Z=53)$ present in our material. Therefore, it is important to confirm the identities of the atoms within the lattice using a complementary technique, high angle annular dark-field aberration-corrected scanning transmission electron microscopy (HAADF-AC-STEM). With HAADF-AC-STEM the intensity of contrast is proportional to $Z^{\alpha}$, where $\alpha$ depends on the detector angle and is typically 1.7-1.8 making it easier to distinguish between two heavy atoms within the same structure. ${ }^{34}$ Indeed, HAADF-AC-STEM images of $\mathrm{Ptl}_{2} @$ @WNT clearly show a cubic lattice (figure 6a), similar to that observed with AC-HRTEM and correlates well with the proposed model of encapsulated $\mathrm{Ptl}_{2}$.

AC-HRTEM images of PtS $\mathrm{S}_{2} @ S W N T$ material prepared in the one-potone-reactant synthesis clearly show extended inorganic nanostructures within the channels of SWNTs (figure 7a). In contrast to the encapsulated $\mathrm{Pt}_{2} @ S W N T$, only platinum atom columns can be individually resolved by AC-HRTEM in this sample due to the significant difference between the atomic numbers of sulfur and platinum. The encapsulated material in $\mathrm{PtS}_{2} @ S W N T$ is characterised by a hexagonal lattice analogous to that of bulk $\mathrm{PtS}_{2}$ (figure $7 \mathrm{~b}$ ). The edge of the encapsulated $\mathrm{PtS}_{2}$ adopts a zigzag conformation, with
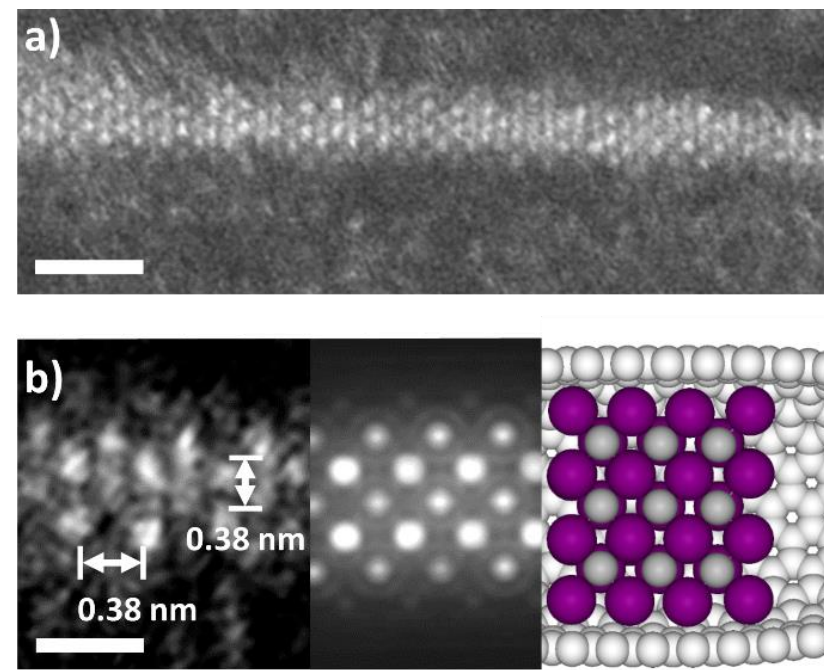

Figure 6: a) HAADF-AC-STEM image showing a SWNT containing $\mathrm{Ptl}_{2}$ in which the SWNT is highly filled with the inorganic material, the scale bar is $1 \mathrm{~nm}$; b)HAADF- AC-STEM micrograph showing a small section of a SWNT containing PtI 2 material and the corresponding atomic distances to the derived structure of $\mathrm{PtI}_{2}$. The material adopts a cubic configuration analogous to that observed in the bright field images in figure 5 , the scale bar is 0.5 $n m$.
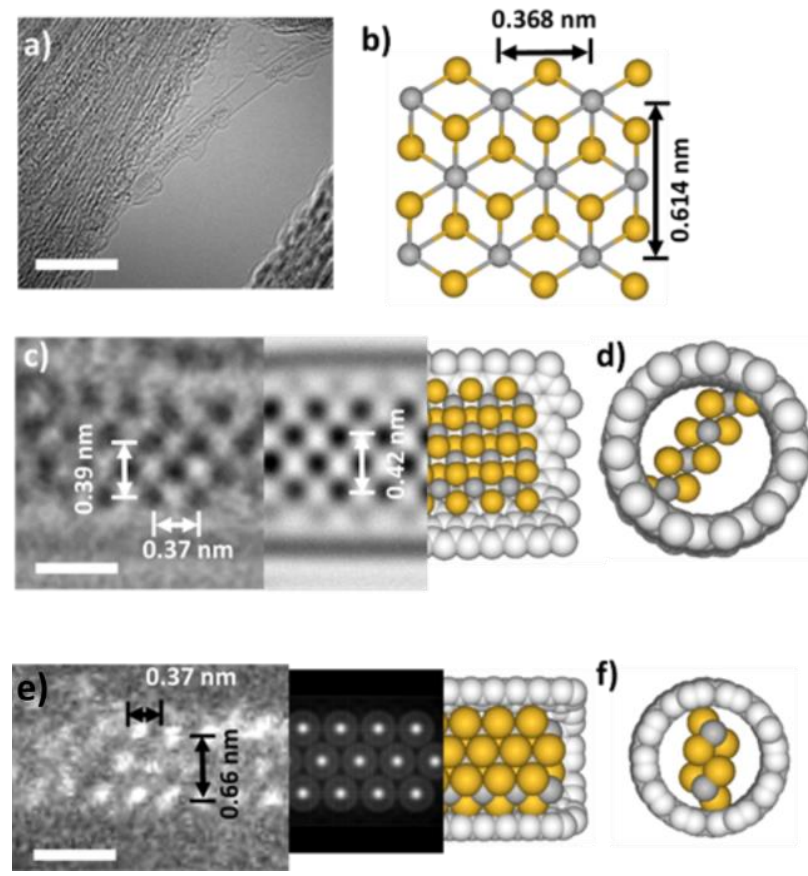

Figure 7: a) An AC-HRTEM micrograph showing a bundle of nanotubes containing PtS $S_{2}$ material. The majority of SWNTS appear to be highly filled, and a single standing tube is visible, the scale bar is $5 \mathrm{~nm}$; b) a portion of the crystal structure of bulk $P t S_{2}$, with key atomic distances shown; the grey and yellow spheres represent platinum and sulfur atoms respectively; c) an AC-HRTEM micrograph showing a small section of a $1.32 \mathrm{~nm}$ SWNT containing PtS $\mathrm{S}_{2}$ and Pt-Pt interatomic distances corresponding to PtS $S_{2}$ structure tilted by $45^{\circ}$ are shown, the scale bar is $0.5 \mathrm{~nm}$; a HR-TEM simulation $(80 \mathrm{kV}$ ) showing how PtS could fit inside a SWNT, complemented by a computational simulation to show its morphology in the nanotube; d) a geometry-optimised model depicting the cross section of PtS,@SWNT; e) a HAADF-AC-STEM image showing what appears to be a single strand of PtS $S_{2}$ in a SWNT, the material adopts a hexagonal configuration analogous to that shown in c); however, there are only three layers of atoms present suggesting that the nanotube is narrower, the scale bar is 0.5 $\mathrm{nm}$. Furthermore, a STEM simulation (200 kV) showing how PtS could fit inside a $(11,11)$ SWNT, complemented by a simulation to show its morphology in the nanotube; f) a geometryoptimised model depicting the cross section of PtS $\mathrm{S}_{2} @ S W N T$.

only sulfur atoms in contact with the nanotube sidewall (Figure 7c,d) and the Pt-Pt separations of the encapsulated $\mathrm{PtS}_{2}$ matching those in the bulk PtS 2 . An HAADF-AC-STEM image of PtS $\mathrm{S}_{2} @ S W N T$ (figure 7e) clearly shows a tilted hexagonal lattice of Pt atoms matching that in AC-HRTEM images for this material (figure 7c). The Pt-Pt spacings appear to be in much closer agreement with bulk $\mathrm{PtS}_{2}$ with only a small increase in the direction parallel to the principal SWNT axis. Although the nanotube sidewall cannot be observed in HAADF-ACSTEM, in contrast to the images obtained using bright field ACHRTEM, only three layers of platinum atoms are present in this sample suggesting that the host nanotube is narrower than in figure 7c (figure 7f). The structural assignment is supported by local probe, quantitative EDX analysis which reveals a Pt:S ratio of 1:1.93, see SI section 5 .

\section{Conclusions}

Inorganic synthesis at the nanoscale is an important, but challenging task because of the need for simultaneous control of the dimensionality and stoichiometry of nanostructures. Carbon nanotubes, acting as nanocontainers effectively provide strict 
control of the size and shape of nanostructures, as we have demonstrated in this study by employing SWNTs as reaction vessels for the direct inorganic synthesis of a series of Pt compounds at the nanoscale. A detailed comparison of the stepwise and one-pot synthetic approaches clearly illustrates the advantages of the latter in terms of efficiency. However, the stepwise approach offers an excellent opportunity for studying the mechanisms of host-guest interactions between the SWNT and encapsulated compounds. We have shown that a combination of FD-XAS and Raman spectroscopy can probe both the inorganic compound and the nanotube properties, and shed light on the direction and magnitude of electron transfer between the nanotube and compound inside. For example, two structurally related $\mathrm{Pt}$ complexes $\mathrm{Pt}(\mathrm{acac})_{2} \mathrm{I}_{2}$ and $\mathrm{Pt}(\mathrm{acac})_{2}(\mathrm{SCN})_{2}$ have shown opposing electronic interactions with SWNTs, withdrawing or donating electron density from/to the hostnanotube, which alters both the oxidation state of the Pt and the vibrational properties of the SWNT. However, neither one-pot nor stepwise approaches appeared to be able to provide sufficient control of the stoichiometry in the inorganic products formed inside the nanotube.

We found a solution to this problem by exploiting the diversity of the coordination chemistry of Pt complexes acting as precursors for the inorganic nanostructures. We expanded the coordination environment of the Pt-atom in the precursor complex by appending extra ligands bearing the necessary elements (I or $S$ ) in a correct and precise proportion to $\mathrm{Pt}$, thus paving the way to stoichiometrically controlled synthesis inside nanotubes, as demonstrated by the synthesis of novel Pt| $\mathrm{Pt}_{2} @ S W N T$ and $\mathrm{PtS}_{2} @ S W N T$ nanostructures in the new one-pot-one-reactant approach with the specifically designed multi-element containing precursors. The new strategy for inorganic synthesis at the nanoscale was shown to be versatile and universal, enabling the formation of previously inaccessible quasi-one-dimensional Pt-containing nanomaterials suitable for a variety of technological applications. ${ }^{35,} 36$ These range from electronic devices where electronic doping of the nanotube is precisely modulated by electron transfer between the SWNT and encapsulated compound, to new catalytic platforms exploiting the unique chemical properties of platinum compounds, where the shape of the crystal and oxidation state of the metal are controlled by steric and electronic interactions with the host-nanotube.

\section{Experimental}

\section{Materials}

SWNTs (P2-SWNTs, arc discharge, Carbon Solutions, USA) were annealed at $600{ }^{\circ} \mathrm{C}$ for $15 \mathrm{~min}$ to open their termini and remove any residual amorphous carbon from their internal cavities resulting in a $50 \%$ weight loss being observed. The platinum precursor, $\mathrm{Pt}(\mathrm{acac})_{2}$ was used as supplied (Sigma Aldrich). The substituted platinum precursors $\mathrm{Pt}(\mathrm{acac})_{2} \mathrm{I}_{2}{ }^{29}$ and $\mathrm{Pt}(\mathrm{acac})_{2}(\mathrm{SCN})_{2}{ }^{30}$ were synthesised in accordance with published procedures.

\section{Ptl $@ S W N T$}

Pt(acac) 2 (90 mg, $0.229 \mathrm{mmol})$ and freshly opened SWNTs $(25 \mathrm{mg}$ ) were sealed together under vacuum $\left(10^{-5} \mathrm{bar}\right)$ in a Pyrex ampoule and heated at $150{ }^{\circ} \mathrm{C}$ for 3 days to ensure penetration of the SWNT by the Pt(acac) 2 . The ampoule was cooled rapidly in ice-water and repetitive washing with dichloromethane $(4 \times 25 \mathrm{~mL})$ was performed, followed by filtration to remove any excess material from the exterior of the SWNTs, forming Pt(acac) $)_{2} @ S W N T$. Under an atmosphere of argon, Pt (acac) ${ }_{2} @ S W N T(10 \mathrm{mg})$ was heated at 500 ${ }^{\circ} \mathrm{C}$ for $1 \mathrm{~h}$ and allowed to cool down slowly forming Pt@SWNT. This sample was sealed in a Pyrex ampoule with an excess of $I_{2}$ and heated at $110^{\circ} \mathrm{C}$ for 3 days to give $\mathrm{Ptl}_{2} @ S W N T$.

Alternatively, $\mathrm{Pt}(\mathrm{acac})_{2} \mathrm{I}_{2}(10 \mathrm{mg} 0.0192 \mathrm{mmol})$ was sealed in a Pyrex ampoule under vacuum $\left(10^{-5} \mathrm{bar}\right)$ with freshly opened SWNTs $(5 \mathrm{mg})$ and heated for 3 days at $150^{\circ} \mathrm{C}$. The ampoule was cooled rapidly in ice water and then heated under argon at $500{ }^{\circ} \mathrm{C}$ for $1 \mathrm{~h}$ to give Ptl $1_{2} @ S W N T$

\section{PtS $@$ @WNT}

Argon was passed over $\mathrm{PtI}_{2} @ S W N T$ (5.7 mg) in a tube furnace for 1 h. The sample was then heated in the presence of a moderate $\mathrm{H}_{2} \mathrm{~S}$ gas flow (ca. $15 \mathrm{~mL} / \mathrm{min}$ ) at $550{ }^{\circ} \mathrm{C}$ for $2 \mathrm{~h}$. The sample was then allowed to cool by $5^{\circ} \mathrm{C} / \mathrm{min}$ under argon.

Alternatively, $\mathrm{Pt}(\mathrm{acac})_{2}(\mathrm{SCN})_{2}(10 \mathrm{mg}, 0.0196 \mathrm{mmol})$ was sealed under vacuum $\left(10^{-5} \mathrm{bar}\right)$ in a Pyrex ampoule with SWNTs $(5 \mathrm{mg})$ and heated at $130{ }^{\circ} \mathrm{C}$ for 3 days. After rapidly cooling in ampoule in ice water, the sample was washed with chloroform $(4 \times 10 \mathrm{~mL})$, then heated under argon at $500{ }^{\circ} \mathrm{C}$ for $1 \mathrm{~h}$ to give $\mathrm{PtS}_{2} @ S W N T$.

\section{Electron Microscopy}

SWNTs filled with the encapsulated materials were dispersed in propan-2-ol using an ultrasonic bath and drop cast onto lacey carbon-coated copper TEM grids (Agar). HRTEM imaging was carried out on a JEOL 2100 FEG-TEM microscope operated at $200 \mathrm{kV}$. ACHRTEM was performed at the University of Ulm on a $C_{s}$ corrected FEI Titan 80-300 TEM operated at $80 \mathrm{kV}$ with information limit enhancement using reduced extraction voltage. HAADF-AC-STEM was performed at the University of Birmingham using a $200 \mathrm{kV}$ JEOL $2100 \mathrm{~F}$ microscope fitted with a CEOS probe corrector and a HAADF detector.

\section{EDX Spectroscopy}

Local EDX spectra were acquired for samples mounted on TEM grids using an Oxford Instruments INCA X-ray microanalysis system. The electron beam was condensed onto areas of specimens (bulk specimen or nanotube bundles) suspended over holes of the amorphous carbon film.

\section{Raman Spectroscopy}

Raman spectroscopy was performed using a HORIBA Jobin Yvon LabRAM HR spectrometer equipped with an automated $x y z$ stage (Märzhäuser). Spectra were acquired using a $532 \mathrm{~nm}$ laser at $0.2 \mathrm{~mW}$ power, in the range 50 to $4000 \mathrm{~cm}^{-1}$, with 5 seconds and 8 accumulations per spectral window, using a 50x objective and a 300 $\mu \mathrm{m}$ confocal pinhole. To simultaneously scan a range of Raman shifts, a 600 lines $/ \mathrm{mm}$ rotatable diffraction grating along a path length of $800 \mathrm{~mm}$ was employed. Spectra were detected using a SYNAPSE CCD detector (1024 pixels) thermoelectrically cooled to $-60^{\circ} \mathrm{C}$. Before the spectra collection, the instrument was calibrated using the Rayleigh line at $0 \mathrm{~cm}^{-1}$ and a standard $\mathrm{Si}(100)$ reference band at $520.7 \mathrm{~cm}^{-1}$.

\section{Image Simulation}


HR-TEM image simulation was carried out using the multislice program QSTEM. QSTEM uses the Dirac-Fock scattering potential of Rez et al. ${ }^{37} \mathrm{~A}$ fixed number of 20 slices per nanotube were chosen and images were calculated with a sampling of $0.05 \mathrm{~nm}$ per pixel. The aberration coefficients and defocus parameters $d f, C_{\mathrm{S}}, A_{1}$, and $B_{2}$ were set according to the imaging conditions in the specific experiment. The convergence angle was fixed at $0.5 \mathrm{mrad}$. The effect of limited electron dose was emulated by applying noise to the calculated images using a custom-made Monte-Carlo program exploiting the Poisson statistics of electrons. Atomic models of SWNTs were built using a custom-made program taking into account different nanotube chiralities, if determinable in the experimental images. The structural models of the inorganic nanomaterials are based on scaled bulk structures and the complex models (PtX@SWNT, X = Pt, S) are rendered and iteratively fitted to the experimental results.

STEM image simulation was performed using both a kinetic approach (for speed) and frozen phonon multislice method to check for dynamical scattering effect which is found to be insignificant. ${ }^{38,39}$ The simulation was performed at $200 \mathrm{keV}$, with a Cs of $10 \mu \mathrm{m}$, convergence angle of $25 \mathrm{mrad}$, inner collection angles $60 \mathrm{mrad}$, outer collection angle of $160 \mathrm{mrad}$, and a mechanical vibration factor of 0.1 $\AA$ A.

\section{Fluorescence-Detected X-ray Absorption Spectroscopy (FD-XAS)}

All FD-XAS measurements were carried out on the XMaS beamline (BM28) at the ESRF. Samples were deposited onto carbon tape, which was then mounted onto a stainless steel sample holder. FDXAS edge inflection points were obtained by fitting a smoothing spline to the measured spectrum, and taking the first derivative of the fitted spectrum.

\section{Acknowledgements}

C.T.S., T.W.C., G.A.R., A.B., M.W.F., and A.N.K. acknowledge support of the European Research Council (ERC), the Engineering and Physical Science Research Council (EPSRC), and the Nanoscale \& Microscale Research Centre (NMRC) for access to instrumentation. J.B. and U.K. acknowledge support of the German Research Foundation (DFG) and the Ministry of Education and Research of the State of BadenWürttemberg within the SALVE project. Z.Y.L. and J.Y. acknowledge the support of EPSRC grants (EP/G070326/1 and EP/G070474/1). The AC-STEM instrument employed in this research was funded through the Birmingham Science City Project.

\section{References}

1. K. Yanagi, Y. Miyata and H. Kataura, Adv. Mater., 2006, 18, 437.

2. T. Takenobu, T. Takano, M. Shiraishi, Y. Murakami, M. Ata, H. Kataura, Y. Achiba and Y. Iwasa, Nat. Mater., 2003, 2 683.

3. Y. Ren and G. Pastorin, Adv. Mater., 2008, 20, 2031.

4. N. A. Kiselev, A. S. Kumskov, V. G. Zhigalina, N. I. Verbitskiy, L. V. Yashina, A. L. Chuvilin, A. L. Vasiliev, A. A. Eliseev and lop, in 18th Microscopy of Semiconducting Materials Conference, eds. T. Walther and J. L. Hutchison, 2013, vol. 471.
5.

R. R. Meyer, J. Sloan, R. E. Dunin-Borkowski, A. I. Kirkland, M. C. Novotny, S. R. Bailey, J. L. Hutchison and M. L. H. Green, Science, 2000, 289, 1324.

6. J. Sloan, S. J. Grosvenor, S. Friedrichs, A. I. Kirkland, J. L. Hutchison and M. L. H. Green, Angew. Chem.-Int. Edit., 2002, 41, 1156.

7. M. Hulman, P. Costa, M. L. H. Green, S. Friedrichs and H. Kuzmany, in Electronic Properties of Synthetic Nanostructures, eds. H. Kuzmany, J. Fink, M. Mehring and S. Roth, Amer Inst Physics, Melville, 2004, vol. 723, pp. 278.

8. E. Flahaut, J. Sloan, S. Friedrichs, A. I. Kirkland, K. S. Coleman, V. C. Williams, N. Hanson, J. L. Hutchison and M. L. H. Green, Chem. Mater., 2006, 18, 2059.

9. B. C. Satishkumar, A. Taubert and D. E. Luzzi, J. Nanosci. Nanotechnol., 2003, 3, 159.

10. A. Chuvilin, E. Bichoutskaia, M. C. Gimenez-Lopez, T. W. Chamberlain, G. A. Rance, N. Kuganathan, J. Biskupek, U. Kaiser and A. N. Khlobystov, Nat. Mater., 2011, 10, 687.

11. T. W. Chamberlain, J. Biskupek, G. A. Rance, A. Chuvilin, T. J. Alexander, E. Bichoutskaia, U. Kaiser and A. N. Khlobystov, ACS Nano, 2012, 6, 3943.

12. L. Shi, P. Rohringer, K. Suenaga, Y. Niimi, J. Kotakoski, J. C. Meyer, H. Peterlik, M. Wanko, S. Cahangirov, A. Rubio, Z. J. Lapin, L. Novotny, P. Ayala and T. Pichler, Nat. Mater., 2016, 15, 634.

13. A. K. Geim and I. V. Grigorieva, Nature, 2013, 499, 419.

14. C. G. Xu, J. Sloan, G. Brown, S. Bailey, V. C. Williams, S. Friedrichs, K. S. Coleman, E. Flahaut, J. L. Hutchison, R. E. Dunin-Borkowski and M. L. H. Green, Chem. Commun., 2000, 2427.

15. J. Sloan, M. C. Novotny, S. R. Bailey, G. Brown, C. Xu, V. C. Williams, S. Friedrichs, E. Flahaut, R. L. Callender, A. P. E. York, K. S. Coleman, M. L. H. Green, R. E. Dunin-Borkowski and J. L. Hutchison, Chem. Phys. Lett., 2000, 329, 61.

16. E. Philp, J. Sloan, A. I. Kirkland, R. R. Meyer, S. Friedrichs, J. L. Hutchison and M. L. H. Green, Nat. Mater., 2003, 2, 788.

17. L. V. Yashina, A. A. Eliseev, M. V. Kharlamova, A. A. Volykhov, A. V. Egorov, S. V. Savilov, A. V. Lukashin, R. Puttner and A. I. Belogorokhov, J. Phys. Chem. C, 2011, 115, 3578.

18. A. A. Eliseev, M. V. Chernysheva, N. I. Verbitskii, E. A. Kiseleva, A. V. Lukashin, Y. D. Tretyakov, N. A. Kiselev, O. M. Zhigalina, R. M. Zakalyukin, A. L. Vasiliev, A. V. Krestinin, J. L. Hutchison and B. Freitag, Chem. Mater., 2009, 21, 5001.

19. Z. Wang, H. Li, Z. Liu, Z. Shi, J. Lu, K. Suenaga, S.-K. Joung, T. Okazaki, Z. Gu, J. Zhou, Z. Gao, G. Li, S. Sanvito, E. Wang and S. lijima, J. Am. Chem. Soc., 2010, 132, 13840.

20. Z. Wang, K. Zhao, H. Li, Z. Liu, Z. Shi, J. Lu, K. Suenaga, S.-K. Joung, T. Okazaki, Z. Jin, Z. Gu, Z. Gao and S. lijima, J. Mater. Chem., 2011, 21, 171.

21. A. Botos, J. Biskupek, T. W. Chamberlain, G. A. Rance, C. T. Stoppiello, J. Sloan, Z. Liu, K. Suenaga, U. Kaiser and A. N. Khlobystov, J. Am. Chem. Soc., 2016, 138, 8175.

22. Q. Fu, G. Weinberg and D. S. Su, New Carbon Mater., 2008, 23, 17.

23. F. Zhang, F. Jiao, X. L. Pan, K. Gao, J. P. Xiao, S. Zhang and X. H. Bao, ACS Catal., 2015, 5, 1381.

24. A. M. Rao, P. C. Eklund, S. Bandow, A. Thess and R. E. Smalley, Nature, 1997, 388, 257. 
25. L. Grigorian, K. A. Williams, S. Fang, G. U. Sumanasekera, A. L. Loper, E. C. Dickey, S. J. Pennycook and P. C. Eklund, Phys. Rev. Lett., 1998, 80, 5560.

26. S. Kiyohara, H. Takamatsu and K. Mori, Semicond. Sci. Technol., 2002, 17, 1096.

27. V. Baranauskas, B. B. Li, A. Peterlevitz, M. C. Tosin and S. F. Durrant, J. Appl. Phys., 1999, 85, 7455.

28. J. X. Zhao and B. Q. Dai, Mater. Chem. Phys., 2004, 88, 244.

29. P. M. Cook, L. F. Dahl, D. Hopgood and R. A. Jenkins, J. Chem. Soc., Dalton Trans., 1973, 294.

30. A. Grabowski and W. Preetz, Z. Anorg. Allg. Chem., 1987, 544, 95.

31. A. A. Eliseev, L. V. Yashina, N. I. Verbitskiy, M. M. Brzhezinskaya, M. V. Kharlamova, M. V. Chernysheva, A. V. Lukashin, N. A. Kiselev, A. S. Kumskov, B. Freitag, A. V. Generalov, A. S. Vinogradov, Y. V. Zubavichus, E. Kleimenov and M. Nachtegaal, Carbon, 2012, 50, 4021.

32. N. A. Kiselev, R. M. Zakalyukin, O. M. Zhigalina, N. Grobert, A. S. Kumskov, Y. V. Grigoriev, M. V. Chernysheva, A. A. Eliseev, A. V. Krestinin, Y. D. Tretyakov, B. Freitag and J. L. Hutchison, J. Microsc., 2008, 232, 335.

33. G. Thiele, W. Weigl and H. Wochner, Z. Anorg. Allg. Chem., 1986, 539, 141.

34. Z. W. Wang, Z. Y. Li, S. J. Park, A. Abdela, D. Tang and R. E. Palmer, Phys. Rev. B, 2011, 84, 073408.

35. X. Y. Chia, A. Adriano, P. Lazar, Z. Sofer, J. Luxa and M. Pumera, Adv. Funct. Mater., 2016, 26, 4306.

36. H. J. Yin, S. L. Zhao, K. Zhao, A. Muqsit, H. J. Tang, L. Chang H. J. Zhao, Y. Gao and Z. Y. Tang, Nat. Commun., 2015, 6, 8.

37. D. Rez, P. Rez and I. Grant, Acta Crystallogr. Sect. A, 1994, 50, 481.

38. D. S. He, Z. Y. Li and J. Yuan, Micron, 2015, 74, 47.

39. R. Aveyard, R. Ferrando, R. L. Johnston and J. Yuan, Phys. Rev. Lett., 2014, 113, 075501. 